\title{
Corela
}

Cognition, représentation, langage

HS-22 | 2017

Prédication et prépositions en anglais

\section{La prédication en grammaire générative}

\author{
Jean-Charles Khalifa
}

\section{OpenEdition}

\section{Journals}

Édition électronique

URL : http://journals.openedition.org/corela/4925

DOI : 10.4000/corela.4925

ISSN : 1638-573X

\section{Éditeur}

Cercle linguistique du Centre et de l'Ouest - CerLICO

\section{Référence électronique}

Jean-Charles Khalifa, "La prédication en grammaire générative », Corela [En ligne], HS-22 | 2017, mis en ligne le 19 juin 2017, consulté le 30 avril 2019. URL : http://journals.openedition.org/corela/4925 DOI : $10.4000 /$ corela.4925

Ce document a été généré automatiquement le 30 avril 2019

\section{(c) (i) (2)(2)}

Corela - cognition, représentation, langage est mis à disposition selon les termes de la licence Creative Commons Attribution - Pas d'Utilisation Commerciale - Partage dans les Mêmes Conditions 4.0 International. 


\title{
La prédication en grammaire générative
}

\author{
Jean-Charles Khalifa
}

\section{Introduction}

1 Nous voudrions faire deux remarques liminaires : en premier lieu - et pour bien préciser "d'où nous parlons ", comme on disait autrefois dans un certain contexte politique - on dit parfois qu'il existe autant de théories syntaxiques que de syntacticiens, mais nous prendrons ici pour cadre un compromis entre plusieurs versions simplifiées tournant autour de ce que l'on nomme Principles and Parameters, ce qui renvoie également, mais nous n'en dirons rien ici, derrière ce terme de "Principes ", à la notion de Grammaire Universelle (Universal Grammar), déclinée selon les langues naturelles en paramètres spécifiques.

2 En second lieu, disons d'emblée que le domaine de travail du syntacticien est limité, comme l'illustre la citation du célèbre fonctionnaliste Halliday (1985), à la phrase et ne saurait, à quelques exceptions près, être étendue à l'échelle du texte. Pour dire les choses de façon plus brutale, aucune explication de texte ne saurait prendre pour cadre théorique une théorie syntaxique quelconque :

By and large, therefore, the chapters that follow take as their domain the traditional realm of syntax, the terrain from the sentence to the word. Grammatically, that is where the action is ; and within that, the fundamental unit of organization is the clause.

3 Nous allons plutôt aller chercher dans le texte qui nous a été fourni des illustrations de divers aspects du thème à l'étude, à savoir la prédication. Commençons par quelques définitions. 


\section{Quelques définitions}

4

Les théories syntaxiques, "génératives » ou autres, ne présentent guère d'originalité bouleversante dès lors qu'il s'agit de se donner une définition large ${ }^{1}$ de la notion de prédication. Prenons précisément une perspective très large, en l'occurrence une perspective typologique. Chez des auteurs classiques en la matière, par exemple Croft (1991, 2001), cette notion est d'abord définie comme relevant de la pragmatique, plus précisément comme s'inscrivant dans les fonctions «communicatives", et faisant système avec la construction de la référence et la modification :

The act of reference identifies a referent, i.e. an entity that one wants to talk about, and establishes a cognitive file for that referent. The act of predication ascribes something to a referent, i.e. it reports that this referent is involved in some state of affairs. The act of modification enriches either reference or predication, by expressing an additional feature of either a referent or a state of affairs (Croft 2001 : 66).

Ces trois fonctions, référence, prédication et modification peuvent être réalisées par des formes linguistiques de types assez divers, mais la façon la plus simple de voir les choses reste sans doute de les assigner à un syntagme composé d'un seul élément lexical. Prototypiquement, c'est la classe des noms qui sera associée aux actes communicatifs de référence (cf. beach, l.1) ; les adjectifs sont quant à eux, toujours prototypiquement, associés à la modification des expressions référentielles (cf. cloudy sky 1.3); et bien évidemment ${ }^{2}$ la prédication sera associée à la catégorie du verbe; on peut ajouter éventuellement que les adverbes, par exemple, seront également associés à la modification, qu'il s'agisse de modification de prédications verbales (cf. thin kind that easily burns (1.8), ou adjectivales (two entirely different trips 1.6). Car bien évidemment, les associations que nous venons de rappeler (N/ référence, A/ modification, V/ prédication) ne sont que les cas non-marqués, et l'on peut trouver, en anglais comme translinguistiquement, tous les cas de figure, résumés dans le tableau $n^{\circ} 1$, emprunté à Croft (2001) :

\begin{tabular}{|lllr|}
\hline \multirow{2}{*}{ OBJECTS } & REFERENCE & MODIFICATION & PREDICATION \\
& $\begin{array}{l}\text { object reference: } \\
\text { UNMARKED NOUNS }\end{array}$ & $\begin{array}{l}\text { object modifier: } \\
\text { genitive, adjecti- } \\
\text { valizations, } \\
\text { PP's on nouns }\end{array}$ & object predication: \\
& & predicate nominals \\
PROPERTIES & $\begin{array}{l}\text { property reference: } \\
\text { deadjectival nouns }\end{array}$ & $\begin{array}{l}\text { property modifier: } \\
\text { UNMARKED ADJECTIVES }\end{array}$ & property predication: \\
ACTIONS & $\begin{array}{l}\text { action reference: } \\
\text { action nominals, } \\
\text { complements, } \\
\text { infinitives, gerunds }\end{array}$ & $\begin{array}{l}\text { action modifier: } \\
\text { participles, } \\
\text { relative clauses }\end{array}$ & action predication: \\
& & & \\
& & & \\
& & &
\end{tabular}

\section{TABLEAU N ${ }^{\circ} 1$ (CROFT, 2001)}

Regardons donc comment la grammaire générative traite de la prédication verbale, et donc des verbes ${ }^{3}$. Un rapide rappel de l'architecture de base postulée (cf. Jackendoff, 1977) pour tout syntagme, quelle que soit la nature de la tête (d'où l'utilisation de $\mathrm{X}$ pour signifier que toute tête lexicale (nominale, verbale, adjectivale, prépositionnelle, 
adverbiale...) ou fonctionnelle ${ }^{4}$ se projettera de la même façon. La Figure 1 détaille le mécanisme de cette projection, par première fusion (merge) avec un complément, puis par seconde fusion du constituant intermédiaire ainsi obtenu avec un spécifieur ${ }^{5}$ :

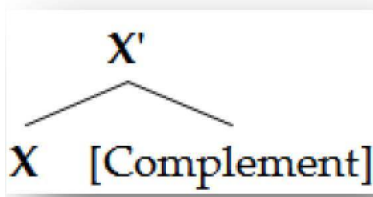

FIg. 1: LE SCHÉMA X-BARRE

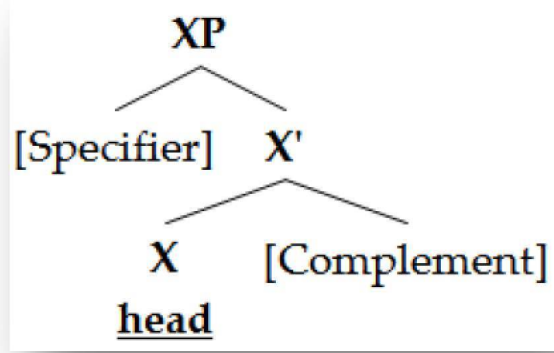

Pour un syntagme verbal, on aura donc :

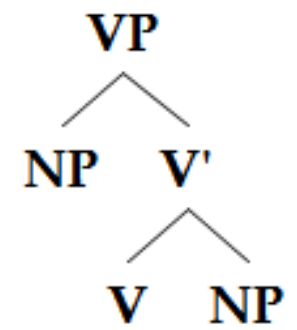

FIg. 2 : SYNTAgME VERBAL (VP) INTRANSITIF

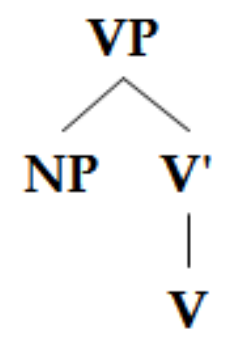

\section{FIg. 3 : VP AVEC V}

8 Autrement dit, nous avons ici un état de la théorie où le VP est réputé ne contenir strictement que le verbe et ses arguments (voir Figure 3 pour un $\mathrm{V}$ intransitif où seul un argument est instancié, et où disparaît donc la position "complément ", représentation qui sera affinée infra). On pourra donc ainsi représenter, par exemple, Macon wore a formal summer suit (1.4) ou bien The car swerved (1. 80), représentations simplifiées données à titre illustratif en Figures 2' et 3' : 


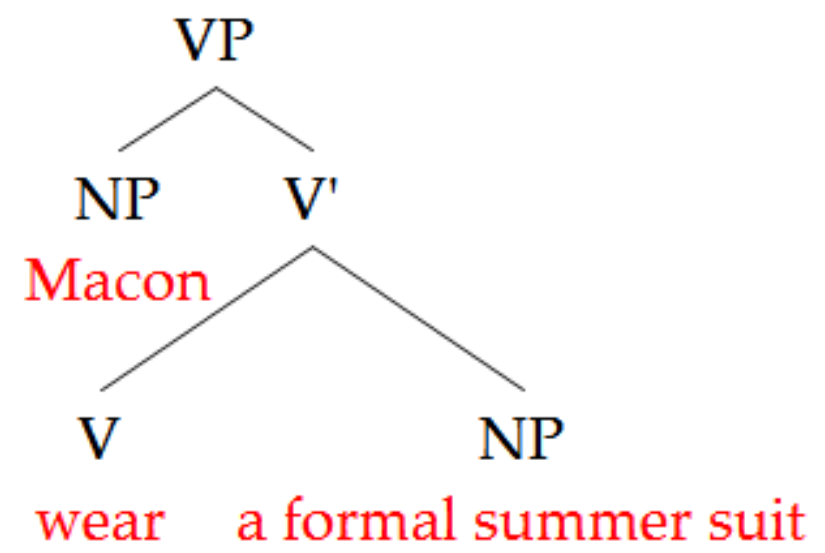

FIg. 2'

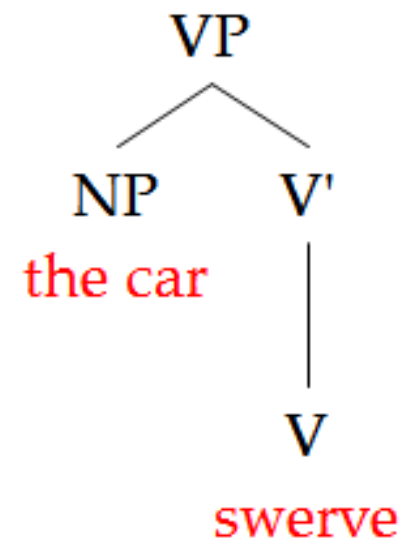

FIg. 3'

Deux remarques cruciales à ce stade de l'exposé : d'une part, la conception sous-jacente du " prédicat » dans les représentations des Figures 2 et 3, dite VPISH (VP-Internal Subject Hypothesis) est de toute évidence frégeienne et non aristotélicienne (voir note 3 ). La grammaire générative, jusqu'à la fin des années 80 , a cependant été dominée par une conceptualisation aristotélicienne, binaire, où sujet s'opposait à prédicat pour constituer la phrase. C'est ce que l'on trouvera en Figure 4 :

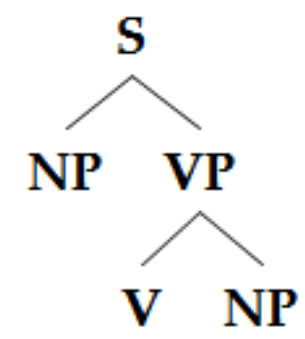

FIg. 4 : REPRÉSENTATION $S \rightarrow$ NP+VP

On aura remarqué, même sans expérience préalable de la syntaxe générative, que jusquelà, il n'est justement pas question de prédication dans les représentations données, car la 
tête verbale est instanciée par le verbe nu, sans aucune marque aspectuo-temporelle. Il s'agit de la «boîte » intérieure, du schéma fondamental d'événement que, selon Carnie (2008), on retrouve sous une forme ou une autre dans toutes les théories syntaxiques, dans ce qu'il nomme un schéma tripartite de la phrase (Figure 5) :

Speaker attitude / Force / Informational structure

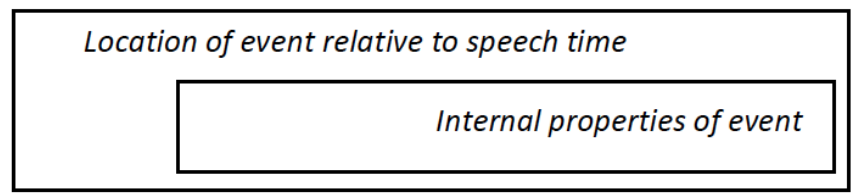

\section{FIg. 5 (CARNIE 2008)}

11 Or, en grammaire générative, le niveau intermédiaire est représenté par une projection fonctionnelle I(nflexion) (voir note 3), dont la tête va accueillir les marques verbales ; en l'occurrence, ici, il ne s'agit que de la marque du passé.

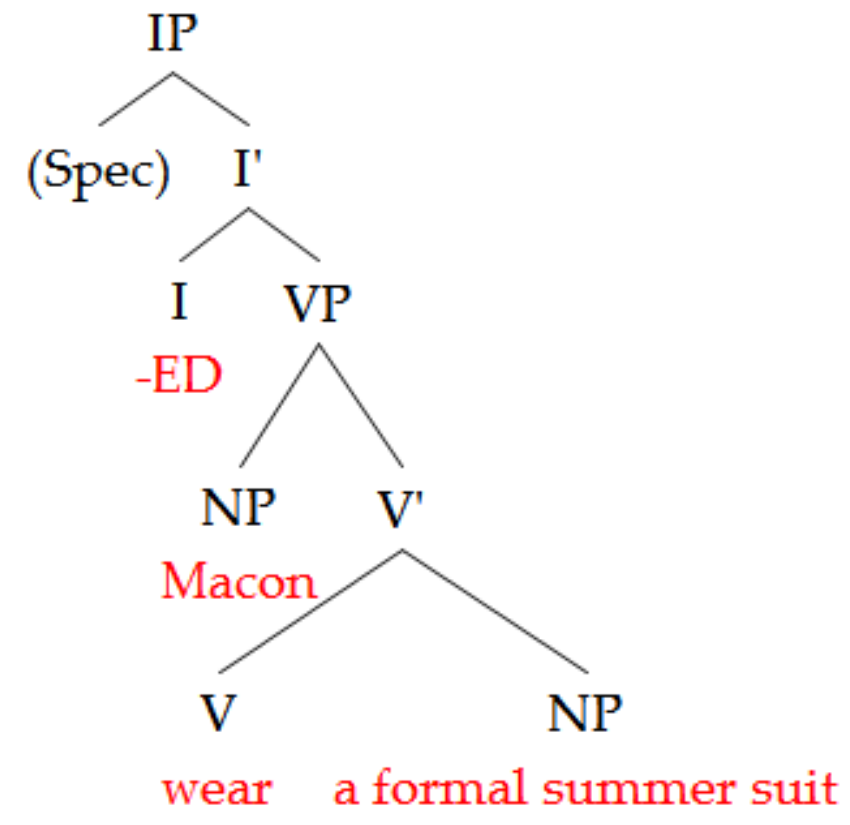

FIg. 2"

Nous ne nous attarderons pas sur la mécanique qui se met en place dès lors, donnée par des principes indépendants contenus dans divers modules de la théorie (rôles sémantiques, attribution des cas, descente de la marque de temps sur le $\mathrm{V}$, ce qui différencie l'anglais, langue à morphologie pauvre, du français, langue à morphologie riche...). Retenons simplement, pour ne pas alourdir inutilement cette présentation, que c'est précisément à ce niveau supérieur (ou, pour utiliser une autre représentation, « la couche intermédiaire " de la Figure 5) que va se jouer la prédication, entendue dans son sens le plus simple et le plus évident: l'événement est désormais muni de ses coordonnées temporelles, et ce qui n'était que l'argument externe du verbe va devenir, à proprement parler, son sujet dès lors qu'il va être marqué du cas nominatif ; pour cela, il faut qu'il quitte sa position de départ et se déplace vers la position [Spec, IP]. Il est 
d'ailleurs frappant de constater les similitudes entre ce déplacement et le traitement de la lexis instanciée en Théorie des Opérations Énonciatives (T.O.E.). On nous pardonnera de nous citer nous-même, mais voici ce que nous écrivions à ce sujet en 2001 :

"Même si l'on retrouve, comme auparavant, un argument «interne » (le NP complément) et un argument " externe» (le NP sujet), cette représentation rend l'analyse beaucoup plus compatible avec la représentation du schéma de lexis, puis de la lexis instanciée, qui n'est rien d'autre que le verbe et ses arguments, puis de la lexis prédiquée, inséparable comme on le souligne souvent de l'opération de thématisation,

'...c'est-à-dire du choix de l'argument susceptible de fournir le thème de l'énoncé. Dans le cas le plus fréquent, à l'actif, c'est le 1er argument qui est choisi comme thème c'est-à-dire posé comme repéré par rapport à la Situation. On peut alors écrire :

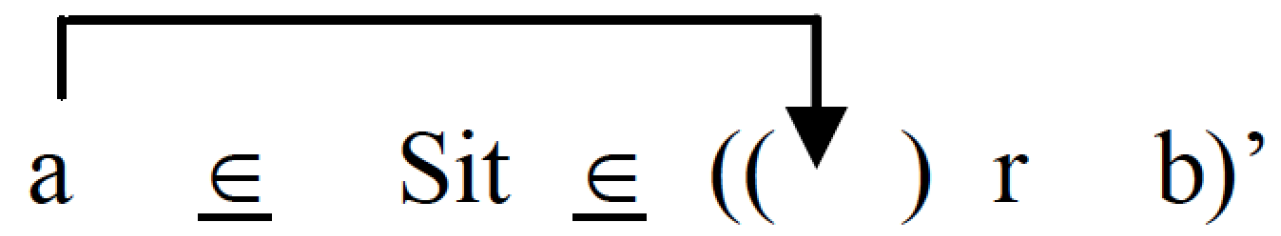

(Groussier \& Rivière 1996, entrée Prédication (opération de)). » (Khalifa, 2001, Document de Synthèse, non publié)

\section{L'ellipse du verbe}

Dès lors que l'on dispose de ces outils d'analyse, un grand nombre de phénomènes liés à la prédication vont pouvoir recevoir une explication dans le cadre théorique sous examen. Regardons par exemple du côté d'énoncés tels que : Sarah had a tan but Macon didn't (l. 6-7), "I don't know that you really care that much," Sarah said. "Do you." (1. 48) et "Well, I just do, sometimes," Sarah said (1. 67). Nous avons en l'occurrence des cas de VP-ellipsis, ou ellipse du Syntagme Verbal. Plus précisément, ce qui se joue dans ces énoncés est l'ellipse du V' (voir supra Figures 2 et 2'), V' qui peut par ailleurs contenir des circonstants en adjonction, comme sometimes (pour l'exemple précédent, on voit bien à la réponse en forme d'interrogation de Macon que that much n'est pas contenu dans le V' ellipsé). Dès lors, seul va être repris le sujet et l'opérateur associé à la prédication, que ce soit Do, comme dans nos trois exemples, ou un autre auxiliaire, comme would dans "Well, I don't know that you would have," (1. 44).

Sans trop développer ce phénomène qui a suscité bien des débats et controverses en syntaxe générative depuis les années 60 , disons que l'on a proposé successivement un certain nombre d'analyses, à commencer par celle de l'effacement (par exemple : Macon didn't have a tan, Do you care? et I just do feel it's my loss alone), puis celle de l'anaphore phonologiquement nulle générée en structure profonde à droite de l'auxiliaire, puis, solution finalement la plus économique, qui est que l'auxiliaire lui-même fonctionne comme une proforme, anaphorisant le V' complet. Dans des analyses plus sophistiquées, le VP-ellipsis est traité comme un cas plus général de mouvement ; tout mouvement en syntaxe est réputé laisser une trace ( $t$ ) dans son site d'origine, et (encore pour simplifier), ces traces doivent être gouvernées par l'auxiliaire. D'où des contrastes comme :

Sarah claimed that..

a. drive carefully, Macon never would t.

b. driven carefully, Macon never had $t$.

c. driving carefully, Macon should be t. 
Sarah claimed that ...

a. *would drive carefully, Macon t.

b. *hasn't driven carefully, Macon t.

c. ?*driving carefully, Macon started t.

d. ?*drive carefully, Macon made her $t$.

où les cas d'agrammaticalité s'expliquent tout simplement par le fait que la trace n'est pas correctement gouvernée. On vérifiera au passage, en manipulant un tant soit peu nos énoncés de départ, que l'ellipse répond exactement aux mêmes contraintes.

L'exemple de la 1.90, quant à lui (You never did like moving), est justiciable d'une autre analyse, sur laquelle nous serons très bref: si nous repartons de la Figure 2", le nœud prédicationnel est sous I, et on observera que, en l'occurrence, c'est un morphème lié (ED) qui occupe cette position; il suffit dès lors de postuler que l'on a tout de même un auxiliaire, en l'occurrence $D o$, phonologiquement nul mais activable dès que le contexte le demande.

\section{Le sujet non exprimé}

Autre phénomène que l'on peut désormais facilement expliquer avec notre boîte à outils, le sujet non exprimé, étroitement lié aux complémentations non finies. Dans l'exemple They decided to come back early (1. 2), To est bel et bien un marqueur de temps, en l'occurrence d'infinitif, et en tant que tel occupera dans la représentation la même position sous I qu'un morphème (libre ou lié) de temps fini ${ }^{6}$. Bien évidemment, cela ne pourra que résonner chez ceux qui travaillent en grammaire méta-opérationnelle, puisque la chose n'est pas sans évoquer l'analyse de Tо comme opérateur de prédication chez Adamczewski (1982)... Par ailleurs, le sujet non exprimé du verbe de l'imbriquée est représenté par PRO, co-indexé au sujet du verbe de l'imbricante, en l'occurrence they (nous n'avons pas fait figurer la coindexation pour le pas alourdir les représentations), puisque, en l'espèce, nous avons affaire à une construction "à contrôle par le sujet » ( subject control). Ce qui nous donne la représentation de la Figure 6 : 


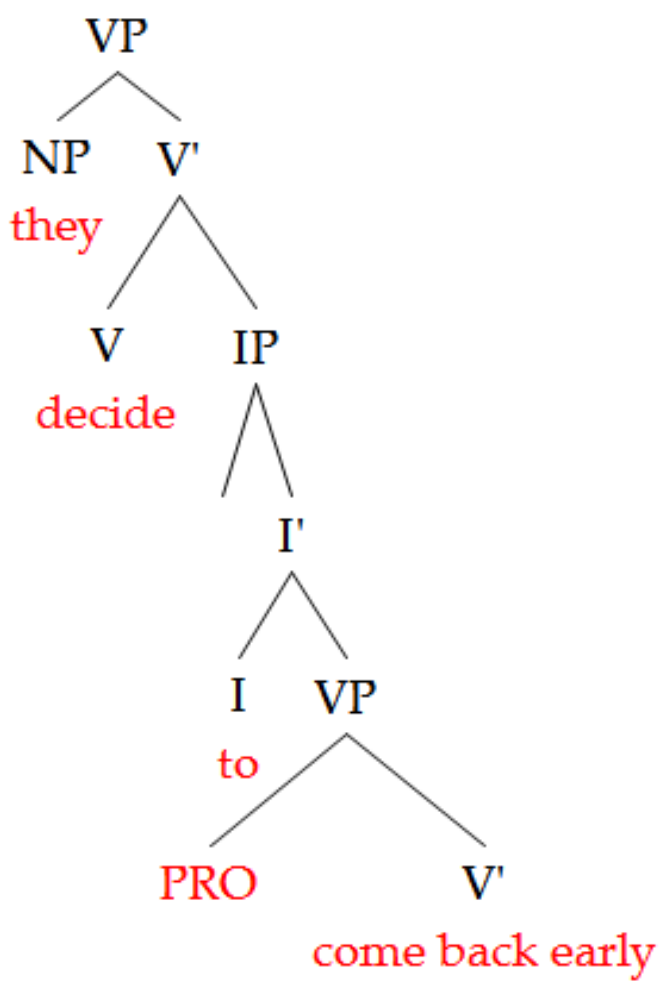

Fig. 6

Notons que beaucoup d'énoncés du texte sont justiciables de la même analyse, avec parfois des raffinements et complexifications; par exemple les extraposées comme It's crazy to ride a motorcycle on a day like today (l. 40), où l'analyse sera la suivante :

$\left[\mathrm{PRO}_{\mathrm{arb}}\right.$ to ride a motorcycle on a day like this] is crazy

PRO est ici dit « arbitraire », car il n'est co-indexé à aucun sujet «contrôleur » dans la phrase. On voit bien par le contexte qu'il renvoie autant au motocycliste qu'à tout sujet potentiel dans la situation. L'analyse reste également valide pour les cas où l'infinitive en то n'est pas argument, mais adjoint, comme I don't know how you can see to drive (1. 24), où l'on postule également la présence d'un sujet PRO, en l'occurrence co-indexé à you.

\section{Enoncés existentiels}

On pourrait aussi, avec cet appareil théorique simplifié (assez rudimentaire, même), analyser les énoncés existentiels en THERE du texte, comme There was a gentle patter on the roof (l.15-16), même si en l'occurrence nous aurions sans doute préféré des locatifs clairs comme There were two students in the corridor. Ces cas mettent bien en évidence tout l'intérêt de faire ce distinguo crucial entre la position d'argument externe (Spec VP) et celle de sujet, étroitement liée à la prédication. En l'occurrence, on aura le NP two students qui, au lieu de monter en Spec IP comme dans le cas par défaut, demeure dans sa position de base, la contrainte subjectale propre à l'anglais, au français et à pas mal d'autres langues étant dès lors satisfaite par l'insertion d'un élément neutre (qui ne porte pas de rôle sémantique), ici THERE (voir Figure 7): 


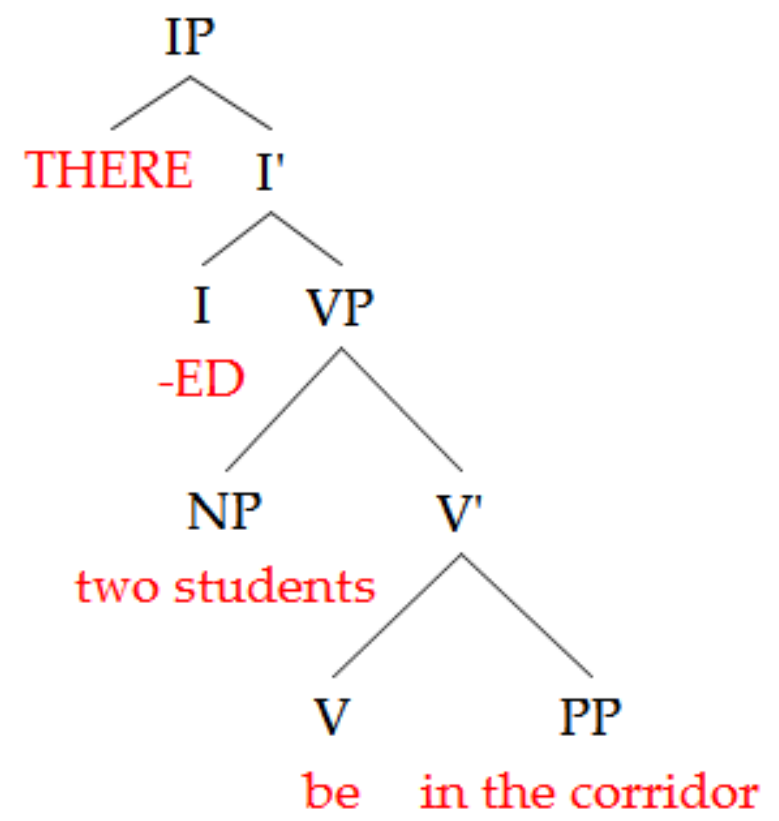

Flg. 7

Notons tout de même que nous serons amenés un peu plus tard à réviser la représentation de la Figure 7, mais pour terminer sur les "explétifs » comme THERE, précisons pour bien compléter les choses que l'on pourrait dire, mutatis mutandis, la même chose du IT de l'extraposée citée supra, ou bien de ceux associés au verbe (à zéro argument) rain : let's hope it doesn't rain (1. 10), it might have been raining for some time (1. 18), de ceux associés à des prédicats tels seem, sur lesquels nous reviendrons infra: it never seemed to me there was all that much point... (1. 53), bref, tous ceux qui ne sont pas référentiels. Nous voyons assez clairement, par conséquent, le lien étroit mis en évidence par la théorie syntaxique entre la contrainte subjectale et la prédication.

\section{Prédicats du type SEEM}

Examinons à présent de plus près les prédicats du type SEEM que nous venons d'évoquer, car ils posent en syntaxe des problèmes fort intéressants; sans entrer dans trop de détails, cette classe de prédicats, dits inaccusatifs, ne prennent qu'un seul argument, mais avec une double particularité par rapport à la représentation des Figures 3 et 3' supra : cet argument est propositionnel et non nominal, et il est réalisé en position postverbale et non préverbale :

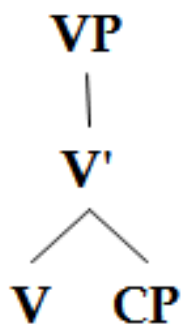


FIg. $8^{7}$

24

Il va dès lors se jouer plusieurs choses: la prédication est impossible en l'état, puisque aucun sujet n'est disponible. Soit on va insérer dans la position requise (Spec, IP), un IT ou un THERE, comme en Figure 7 supra, ce qui peut donner l'exemple de la 1. 53, déjà cité (it never seemed to me there was all that much point...), soit un NP va « monter » de l'intérieur de la proposition qui constitue l'argument unique de SEEM, ce qui est le cas dans the rain seemed to fall in sheets (1.45), où le schéma serait :

the rain SEEM $[(t)$ to fall in sheets $]$

C'est là le fameux mouvement dit de «montée du sujet», l'un des classiques de la grammaire générative. Il est on ne peut plus intéressant pour notre propos, puisque somme toute fall in sheets est prédiqué de the rain (c'est bien le verbe fall qui donne son rôle sémantique au NP the rain, qui est donc son argument), mais ce NP va, en fait, servir de sujet syntaxique à seem, avec lequel il n'entretient pourtant aucun rapport de sélection. Un autre exemple relevant de ce cas de figure, bien qu'un peu différent, est They were supposed to stay at the beach qui ouvre le texte (1.1).

Mais les exemples de SEEM copule it seemed marbled (1. 72) et his nose seemed sharper and whiter (1. 83) sont-ils véritablement différents? Bien entendu, dans le premier cas cette fois IT est bel et bien référentiel, puisqu'il renvoie anaphoriquement à windshield. Mais en fait (nous devons, par manque de place, être très allusif ici) toutes les études en la matière dans la littérature générativiste aboutissent à une structure qui ne ressemble pas à une construction transitive classique, celle que l'on trouve par exemple en figure 2" supra, mais bien plutôt à celle de la Figure 8 supra; en l'occurrence :

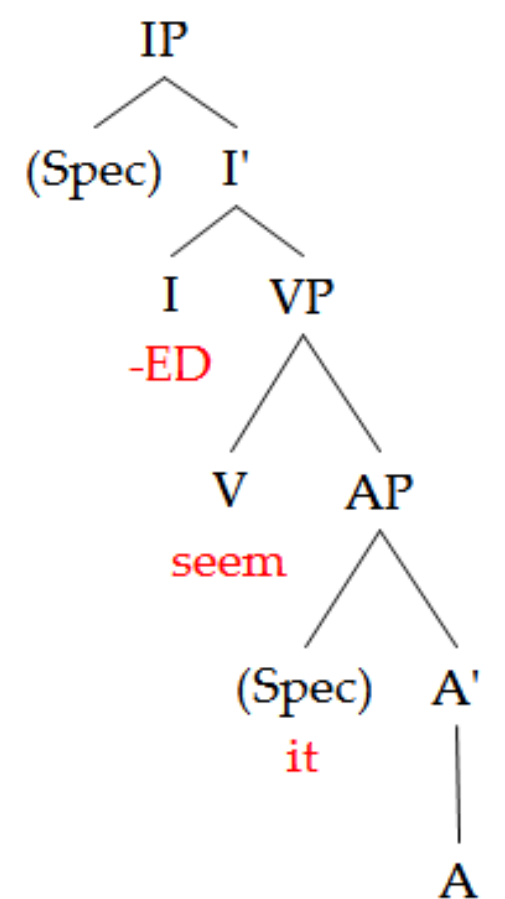

marbled 
FIg. $9^{8}$

En d'autres termes, ce que montre le diagramme ci-dessus, c'est un événement de type propriété, où marbled est associé à it ( = the windshield), le tout étant l'argument unique de seem, qui associé à la marque de temps finie, va dénoter à la fois la prédication d'existence de l'événement en question, et (mais la syntaxe pure ne nous dit rien làdessus, tout au moins pas si l'on s'en tient à ces modèles simplifiés) sa modalisation, de type épistémique, dont on peut même récupérer, pour emprunter des concepts de la T.O.E à nouveau, la « source modale » (par exemple it seemed marbled to her).

à étendre cette analyse de SEEM copule comme verbe à montée (raising verb) à toutes les occurrences de $B E$ copule, qui abondent dans le texte, il n'y a qu'un pas, que nous franchirons pour conclure cette étude. On sait en effet depuis très longtemps que la copule n'a pas les mêmes propriétés syntaxiques que les verbes lexicaux, mais se rapproche davantage des auxiliaires. Par exemple, pour reprendre les NICE-properties de Huddleston (1976), elle apparaît à gauche de la négation, laquelle peut se cliticiser sur elle, elle s'inverse avec le sujet et apparaît à gauche de l'adverbe, etc. Et, bien entendu, les contraintes et restrictions sémantiques sur le sujet sont imputables exclusivement au syntagme complément (NP, AP, PP), et non à la copule elle-même. La seule caractéristique qu'elle partage avec les verbes lexicaux est qu'elle ne peut être suivie d'une autre catégorie verbale?.

Ce que l'on peut dire très rapidement, au plan syntaxique, c'est que la prédication avec $B E$ copule revient finalement à poser l'existence d'un prédicat nominal (par exemple a tall, pale, gray-eyed man, 1. 7), ou bien adjectival (dead, 1. 50) muni de son argument (respectivement he et Ethan); ce qui en gros se représente comme suit :

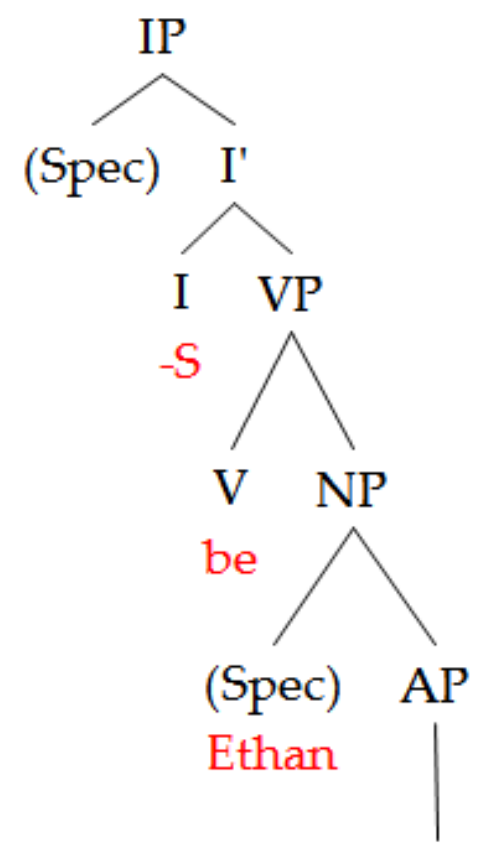

A

dead 


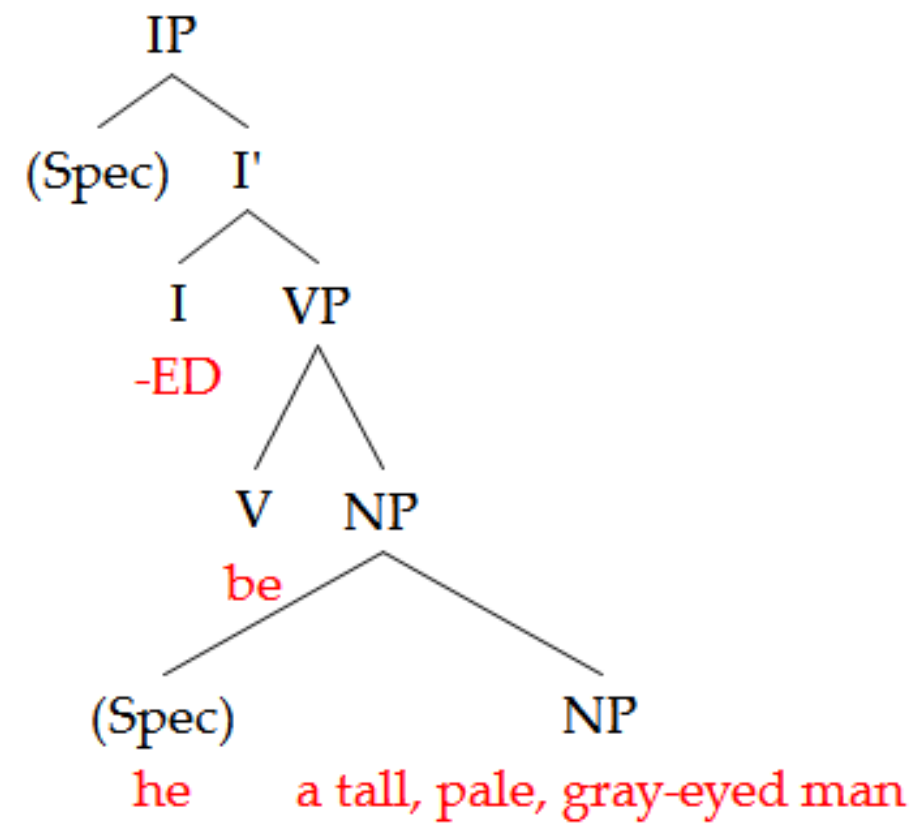

FIg. 11

\section{Conclusion}

De ce bref tour d'horizon, on pourra utilement retenir, au-delà des aspects techniques de la théorie dans le cadre de laquelle nous nous situons, les rapprochements et recoupements, parfois évidents, parfois plus ou moins cachés, avec les autres approches mises en miroir dans le présent ouvrage. Nous avons toujours soutenu, parfois contre vents et marées, que les théories ne pouvaient être antagonistes, mais qu'elles devaient toujours être prises comme complémentaires et mutuellement enrichissantes. Nous espérons que le lecteur tirera profit de cette confrontation, qui dans notre esprit, on l'aura compris, n'est nullement à prendre comme une compétition.

\section{BIBLIOGRAPHIE}

Adamczewski, H. (1982). Grammaire linguistique de l'anglais. Paris : Armand Colin.

Aristote (1994). Catégories. De l'interprétation. Organon I-II. Paris : Vrin

Baker M. (2003). Lexical Categories: Verbs, Nouns and Adjectives, Cambridge Studies in Linguistics, Cambridge University Press.

carnie A. (2008). Constituent Structure, Oxford : Oxford University Press. 
Croft w. (1991). Syntactic Categories and Grammatical Relations : The Cognitive Organization of Information , Chicago :University of Chicago Press.

croft W. (2001), Radical Construction Grammar : Syntactic Theory in Typological Perspective, Oxford : Oxford University Press.

Frege G. (1971). Ecrits Logiques et Philosophiques. Paris : Seuil.

Halliday M.A.K. (1985). Introduction to Functional Grammar, London : Arnold.

Huddleston R. (1976). 'Some Theoretical Issues in the Description of the English Verb', Lingua, 40, 4.

Jackendoff R. (1977). X-bar Syntax : A Study of Phrase Structure. Cambridge, MA : MIT Press.

Khalifa J.-C. (2001). « Etudes sur l'énoncé complexe ». Document de synthèse pour le diplôme d'Habilitation à Diriger des Recherches, Université de Poitiers (document non publié).

Khalifa J.-C. et Miller P. (2009). 'TO and TO-Clauses in Formal Grammars', Publications en ligne de l'ALAES. https://alaesfrance.wordpress.com/ (site en cours de mise à jour)

Merle J.-M. (ce volume). « La prédication. Approche des opérations prédicatives et énonciatives » williams E. (1980). 'Predication', Linguistic Inquiry, 11, 1, p. 203-238.

\section{NOTES}

1. La précision est utile, car les choses sont bien différentes lorsque l'on tente de se donner une définition étroite. En l'occurrence, le lecteur intéressé pourra consulter l'article fondateur de Williams (1980), où les relations de prédication sont définies en termes de relations structurales, et plus précisément de c-command et de c-subjacency. Nous n'avons pas voulu, dans le cadre de ce travail inter-théorique, entrer dans des considérations aussi techniques.

2. Si, à l'instar de Baker, on exclut les prépositions des grandes «parties du discours" (voir le titre même de son ouvrage de 2003 : Lexical Categories: Verbs, Nouns, and Adjectives). Cela est un autre débat, qui ne nous concerne pas ici.

3. Un mot tout de même pour lever un certain nombre de confusions assez classiques et à notre sens fâcheuses. Il existe dans la littérature au moins deux sens à "prédicat", que nous nommerons respectivement « Aristotélicien » et «Fregéien ». Dans le premier cas, le prédicat est ce qui reste de la phrase quand on en a retranché le sujet (cette conception binaire de la phrase comme composée d'un sujet et d'un prédicat remonte en effet à Aristote (384-322 av. JC) cf. Aristote (1994); et par conséquent la prédication est la mise en relation d'un sujet et d'un prédicat entendu dans ce sens ; par exemple, Rain // flattened the long, pale grass at the sides of the road (1. 16-17). Dans le second cas, le prédicat se laisse réduire à un seul lexème ; cette conception, bien plus récente, peut être attribuée au mathématicien et philosophe Frege (1848-1925), l'un des fondateurs de la logique moderne. Ainsi, dans la même phrase du texte, on dira que le prédicat est FLATTEN, et ses deux arguments RAIN et GRASS. Mais pour une étude plus détaillée, voir la contribution de Merle dans le présent recueil.

4. Jackendoff n'utilisait pas encore les têtes fonctionnelles (par exemple I(nflection), C (omplementizer), etc.), qui sont introduites dans les diverses versions de la théorie à compter des années 80 . Mais même lorsque la tête est fonctionnelle, l'architecture est postulée identique, avec les deux fusions successives (X-complément et $\mathrm{X}$ '-spécifieur) pour donner le niveau syntagmatique (XP). 
5. Deux remarques au passage : " complément » et « spécifieur » sont des positions structurales, et sont optionnelles, au sens où elles peuvent ne pas être instanciées.

6. Notons qu'il ne s'agit là que d'une des conceptions - même si elle est largement dominante, en particulier dans les manuels de syntaxe - qui se sont succédé quant à la nature et la position de TO. Pour plus de précisions et une étude exhaustive, on pourra se reporter à Khalifa \& Miller (2009, disponible sur le site de l'ALAES).

7. $C P=$ Complementizer Phrase

8. AP = Adjective $; A P=$ Adjective Phrase

9. Si nous revenons un cran en arrière, nous constaterons que les propriétés syntaxiques de SEEM, par exemple, s'alignent sur celles de $B E$, à l'exception de la cliticisation et de la position par rapport à l'adverbe.

\section{RÉSUMÉS}

Cette étude se veut une synthèse des présentations de la notion de prédication dans le cadre général des Principes et Paramètres en grammaire générative. On montre tout d'abord que la prédication est étroitement liée, au plan typologique, au verbe, et que plus précisément dans le cadre retenu, c'est au niveau de la catégorie fonctionnelle I ( = Inflection), qui domine le groupe verbal, que se joue la prédication, cette catégorie étant également étroitement liée à la notion de sujet. Dès lors, à partir des exemples du texte, on examinera successivement les divers cas de figure pertinents, dont les structures existentielles-présentatives en there et les inaccusatifs comme seem, auxquels on montrera que s'assimilent les be copule.

In this paper, we try to synthesize the various approaches to predication under the Principles \& Parameters framework. It is first shown that, from a typological point of view, predication is closely linked to the verb, and more precisely to the functional projection I ( = Inflection) that dominates the VP. This particular layer is also closely linked to subjecthood. Starting from the examples taken in the text, we then go on to discuss the relevant cases, including there existential-presentative constructions and inaccusative predicates like seem, which will be shown to cover be copular structures.

\section{INDEX}

Mots-clés : prédication, principes et paramètres, projection fonctionnelle, flexion verbale, inaccusativité.

Keywords : predication, functional projection, inflection, inaccusativity. 


\section{AUTEUR}

\section{JEAN-CHARLES KHALIFA}

Université de Poitiers

Forell-Cerlitep (EA 3816)

jean.charles.khalifa@univ-poitiers.fr 